\title{
Influence of Span Ratio on Modals and Mode Direction Factors of Curved Box-girder Bridges
}

\author{
Guohua Song ${ }^{1}$, Dongwei Wang ${ }^{1}$ and Xiaofang Liu ${ }^{2}$ \\ ${ }^{1}$ School of Civil Engineering, Zhengzhou University, Zhengzhou 450001, China \\ ${ }^{2}$ Urban and Ural Construction and Designing Institute of Henan Province Co. Ltd, Zhengzhou 450004, China
}

\begin{abstract}
The vibration mode of curved box-girder bridge was decomposed into 4 mode direction factors by energy ratios. 4 twospan curved box-girder bridge models were built taking span ratio as parameter. The modal characteristics and the mode direction factors were analyzed along with varying span ratio. It is advised that the stiffness increases more slowly than mass for curved box-girder bridge, the vertical bending mode must be taken account, the torsion mode factor decreases with the decreasing of span ratio, the summit of longitudinal movement direction mode factor appears on higher modal orders, and high order modals must be considered when designing.
\end{abstract}

Keywords-span ratio; curved box-girder bridges; modal; mode direction factor

\section{INTRODUCTION}

China is prone to earthquake disasters in the world. It is very important for bridges on road transport to have earthquake resistance in research of disaster prevention and reduction. Many curved box-girder bridges are built in high grade highways, urban overpasses, and viaducts. Recently, influence factors of static and dynamic performances and seismic response laws about curved box-girder bridges have been extensively studied ${ }^{[1-3]}$. The influence factors mainly include buckling, distortion, shear lag effect, initial curvature, supporting form, curvature radius, span ratio, ratio of depth to span, and ratio of span to width etc ${ }^{[4]}$. During vibration of a system, the kinetic energy and the potential energy can be converted each other for every same order of vibration mode, but energy cannot be converted between different mode $\operatorname{orders}^{[5]}$.

Vibration modes have three types: basic modes, coupled modes, and local modes. Basic modes include translation mode, bending mode, and torsion mode; coupled modes are supervised by more than two basic modes; and local modes are basic modes generated by structural members with weak stiffness or weak joints ${ }^{[6,7]}$.

If the vibration modes of curved box-girder bridges are decomposed into four sub directions as vertical bending, pure torsion, lateral bending, and longitudinal movement, and vibration mode is one sub-direction-based, the sub direction will be defined as principal direction of the curved box-girder bridge ${ }^{[8]}$. It is observed that it is very difficult to decide the principal direction of curved box-girder bridge only by the shape or the feature vector. In reference [8] four direction parameters were used to express the ratio of sub direction energy to total energy of every vibration mode, the value of direction parameter will decide the principal direction of each vibration mode.

\section{DiRECTION FACTORS OF VIBRATION MODE}

From the energy point, the energy $W_{j}$ of every order vibration mode of element $j$ is decomposed into energy in four sub-directions: the vertical bending energy $\left(W_{j}\right)_{\mathrm{vb}}$, the lateral bending energy $\left(W_{j}\right)_{\mathrm{lb}}$, the torsion energy $\left(W_{j}\right)_{\mathrm{t}}$, and the longitudinal movement energy $\left(W_{j}\right)_{\operatorname{lm}}$, that is,

$$
W_{j}=\left(W_{j}\right)_{\mathrm{vb}}+\left(W_{j}\right)_{\mathrm{lb}}+\left(W_{j}\right)_{\mathrm{t}}+\left(W_{j}\right)_{\mathrm{lm}}
$$

Define $\alpha_{j}$ as the ratio of $\left(W_{j}\right)_{\mathrm{vb}}$ to $W_{j}$, which is named vertical bending direction factor; Define $\beta_{j}$ as the ratio of $\left(W_{j}\right)_{\mathrm{lb}}$ to $W_{j}$, which is named lateral bending direction factor; Define $\gamma_{j}$ as the ratio of $\left(W_{j}\right)_{\mathrm{t}}$ to $W_{j}$, which is named torsion direction factor; Define $\delta_{j}$ as the ratio of $\left(W_{j}\right)_{\operatorname{lm}}$ to $W_{j}$, which is named longitudinal movement direction factor. The four factors $\alpha_{j}, \beta_{j}, \gamma_{j}$, and $\delta_{j}$ are expressed as percentage.

The curved box-girder bridge model is divided into $n$ nodes and $(n-1)$ elements, irrespective of the bulking deformation; every node has 6 freedoms, which are $u_{x}, u_{y}, u_{z}, \theta_{x}, \theta_{y}$ and $\theta_{z}$. Among them, $u_{x}, u_{y}, u_{z}$ are the translation displacements of cross section in direction of $x, y$, and $z$, respectively; $\theta_{x}, \theta_{y}$ and $\theta_{z}$ are the torsion angles of cross section around $x, y, z$ axis, respectively.

From above definitions, the four direction factors of the $j$ th vibration mode of curved box-girder bridges are expressed as follows: the vertical bending direction factor $\alpha_{j}$, the lateral bending direction factor $\beta_{j}$, the torsion direction factor $\gamma_{j}$, and the longitudinal movement direction factor $\delta_{j}$, they are expressed in formula (2) to formula (5), respectively. 


$$
\begin{aligned}
& \alpha_{j}=\frac{\left(W_{j}\right)_{\mathrm{vb}}}{W_{j}} \times 100 \% \\
& =\frac{\sum_{i=1}^{n}\left(J_{y i} \cdot \theta_{y i j}^{2}\right)+\sum_{i=1}^{n}\left(m_{i} \cdot u_{z i j}^{2}\right)}{\left[\sum_{i=1}^{n}\left(m_{i} \cdot u_{z i j}^{2}\right)+\sum_{i=1}^{n}\left(J_{y i} \cdot \theta_{x i j}^{2}\right)\right]+\left[\sum_{i=1}^{n}\left(J_{x i} \cdot \theta_{x i j}^{2}\right)\right]+\left[\sum_{i=1}^{n}\left(m_{i} \cdot u_{y i j}^{2}\right)+\sum_{i=1}^{n}\left(J_{z i} \cdot \theta_{z i j}^{2}\right)\right]+\left[\sum_{i=1}^{n}\left(m_{i} \cdot u_{x i j}^{2}\right)\right]}
\end{aligned}
$$

$\beta_{j}=\frac{\left(W_{j}\right)_{\mathrm{lb}}}{W_{j}} \times 100 \%$

$=\frac{\sum_{i=1}^{n}\left(J_{z i} \cdot \theta_{z i j}^{2}\right)+\sum_{i=1}^{n}\left(m_{i} \cdot u_{y i j}^{2}\right)}{\left[\sum_{i=1}^{n}\left(m_{i} \cdot u_{z i j}^{2}\right)+\sum_{i=1}^{n}\left(J_{y i} \cdot \theta_{x i j}^{2}\right)\right]+\left[\sum_{i=1}^{n}\left(J_{x i} \cdot \theta_{x i j}^{2}\right)\right]+\left[\sum_{i=1}^{n}\left(m_{i} \cdot u_{y i j}^{2}\right)+\sum_{i=1}^{n}\left(J_{z i} \cdot \theta_{z i j}^{2}\right)\right]+\left[\sum_{i=1}^{n}\left(m_{i} \cdot u_{x i j}^{2}\right)\right]}$

$\gamma_{j}=\frac{\left(W_{j}\right)_{\mathrm{t}}}{W_{j}} \times 100 \%$

$=\frac{\sum_{i=1}^{n}\left(J_{x i} \cdot \theta_{x i j}^{2}\right)}{\left[\sum_{i=1}^{n}\left(m_{i} \cdot u_{z i j}^{2}\right)+\sum_{i=1}^{n}\left(J_{y i} \cdot \theta_{x i j}^{2}\right)\right]+\left[\sum_{i=1}^{n}\left(J_{x i} \cdot \theta_{x i j}^{2}\right)\right]+\left[\sum_{i=1}^{n}\left(m_{i} \cdot u_{y i j}^{2}\right)+\sum_{i=1}^{n}\left(J_{z i} \cdot \theta_{z i j}^{2}\right)\right]+\left[\sum_{i=1}^{n}\left(m_{i} \cdot u_{x i j}^{2}\right)\right]}$

$$
\begin{aligned}
& \delta_{j}=\frac{\left(W_{j}\right)_{\mathrm{lm}}}{W_{j}} \times 100 \% \\
& =\frac{\sum_{i=1}^{n}\left(m_{i} \cdot u_{x i j}^{2}\right)}{\left[\sum_{i=1}^{n}\left(m_{i} \cdot u_{z i j}^{2}\right)+\sum_{i=1}^{n}\left(J_{y i} \cdot \theta_{x i j}^{2}\right)\right]+\left[\sum_{i=1}^{n}\left(J_{x i} \cdot \theta_{x i j}^{2}\right)\right]+\left[\sum_{i=1}^{n}\left(m_{i} \cdot u_{y i j}^{2}\right)+\sum_{i=1}^{n}\left(J_{z i} \cdot \theta_{z i j}^{2}\right)\right]+\left[\sum_{i=1}^{n}\left(m_{i} \cdot u_{x i j}^{2}\right)\right]}
\end{aligned}
$$

Where, $m_{i}$ is the translation mass of node, and $m_{i}=A_{i} \cdot \rho_{i}, \rho_{i}$ is the mass of unit area, $A_{i}$ is node area; $J_{x}$, $J_{y}, J_{z}$ are rotating mass moment of inertia of node $i$ respectively, and $J_{x}=\rho_{i} \cdot I_{x}, J_{y}=\rho_{i} \cdot I_{y}, J_{z}=\rho_{i} \cdot I_{z}$. $I_{x}$ is the torsion inertial moment around the mass center of nodal section of $i, I_{y}, I_{z}$ are the bending inertia moments around notes $y, z$, respectively.

And another condition is,

$$
\alpha_{j}+\beta_{j}+\gamma_{j}+\delta_{j}=100 \%=1
$$


It can be seen from formula (1) through (5) that, the values of direction factors of every modal only relate to structural mass, geometric characteristics of section and modal vectors.

\section{BUILdING Finite ElEMENT Models}

The shape and dimensions of section of modes were determined by the general design and principals of curved boxgirder bridges, the sectional dimensions of models are shown in FIGURE I, the concrete was used C40 grade. The curved boxgirder bridge models were built with two spans, one span was $30 \mathrm{~m}$, and another span was 30, 45, 60 and $75 \mathrm{~m}$, respectively, whose span ratios were $1: 1,1: 1.5,1: 2,1: 2.5$, respectively. Inner supports at two ends of models were constrained in $y$ and $z$ directions, outer supports were constrained in $z$ direction, and middle supports were constrained in $x, y, z$ directions. The finite element model and constraint diagram are shown in FIGURE II. The models were analyzed by finite element software to get modals, and Lanczos method was used to compute eigenvalues.

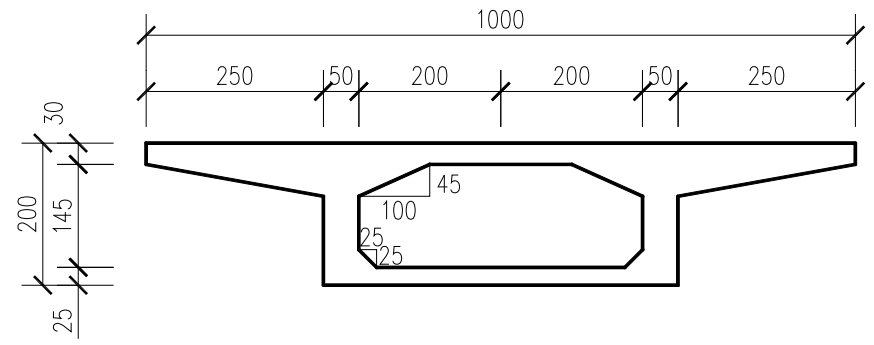

FIGURE I. CROSS SECTION OF MODELS (UNIT: MM)

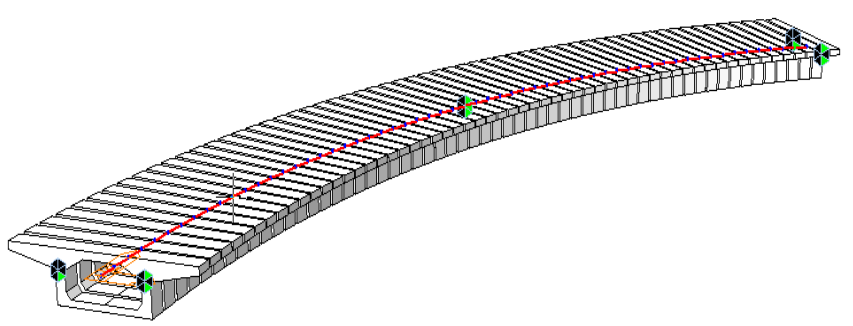

FIGURE II. FINITE ELEMENT MODEL AND CONSTRUCTIONS

\section{MODEL ANALYZING}

\section{A. Influence of Span Ratio on Frequencies of Curved Box-} Girder Bridges

"Guidelines for Seismic Design of Highway Bridges (JTG/T B02-01-2008)" stipulates: The modal orders considered should gain more than 90 percent effective mass in that direction when calculating with multiple modal response spectrum analysis method ${ }^{[9 \sim 11]} .24$ modal orders were analyzed considering effective mass of four models in the paper comprehensively.

The frequency curves of first 24 orders of four models with different span ratios are shown in FIGURE III. As can be seen from FIGURE III that no overlap exits among the frequency curves, in other words, changes of span ratios within certain range have great influence on vibration performance of curved girder bridges. The value of every natural frequency of vibration decreases as the span ratios decrease for curved girder bridges. This means the stiffness of curved girder bridges increases less than the mass increases, or the smaller the span ratio is, the softer the stiffness is.

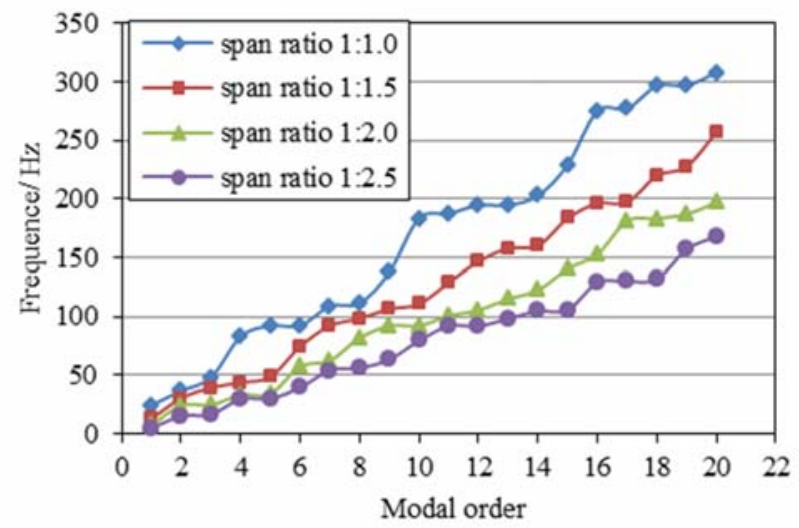

FIGURE III. COMPARISON OF MODAL FREQUENCIES OF MODELS WITH DIFFERENT SPAN RATIOS

\section{B. Influence of Span Ratio on Mode Direction Factors}

Four Mode direction factors of every models were calculated based on formula (2) through (5), and the same mode direction factors of different models were compared, respectively, the results are shown in FIGURE IV to FIGURE VII. 


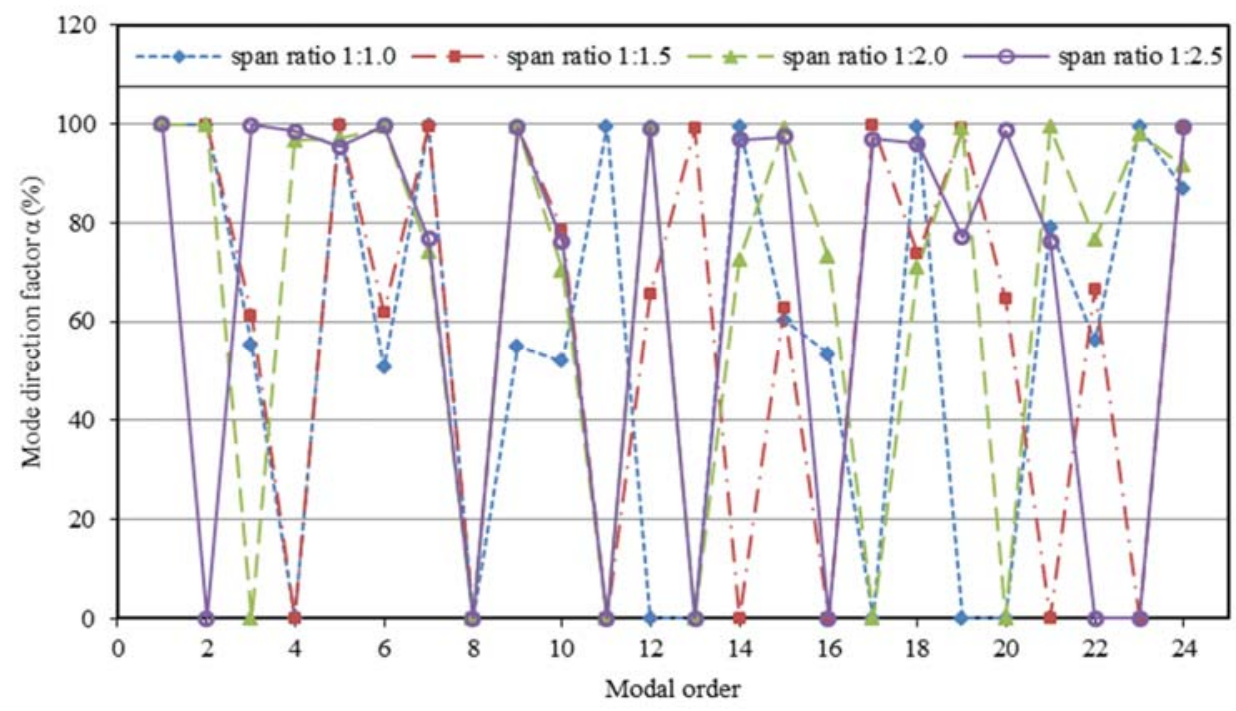

FIGURE IV. VALUES AND COMPARISON OF MODE DIRECTION FACTOR A

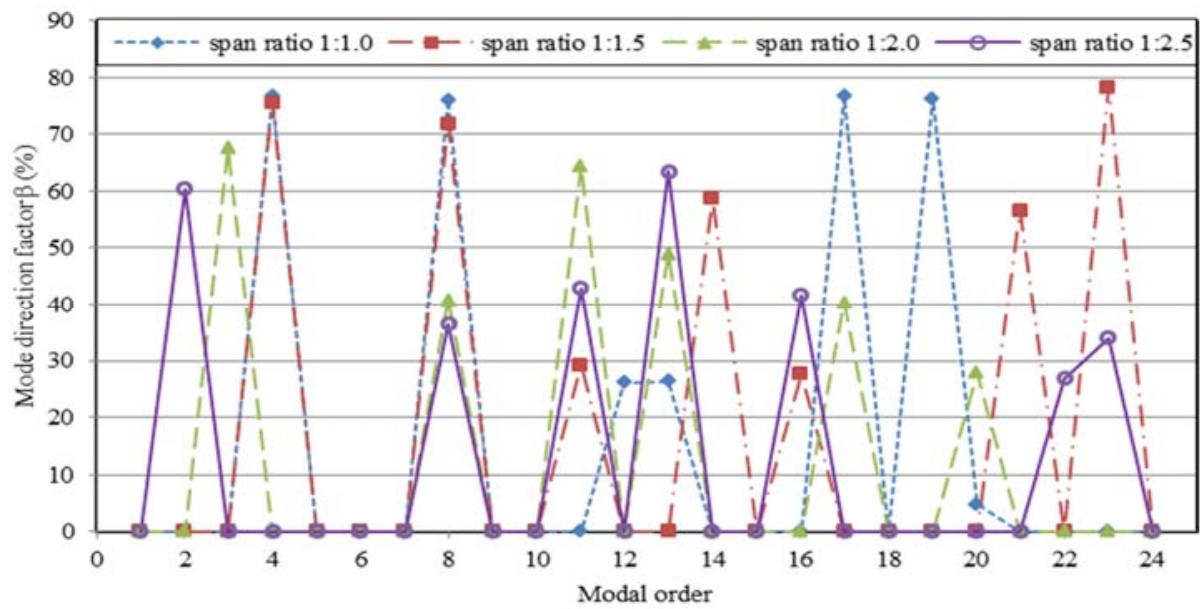

FIGURE V. VALUES AND COMPARISON OF MODE DIRECTION FACTOR B

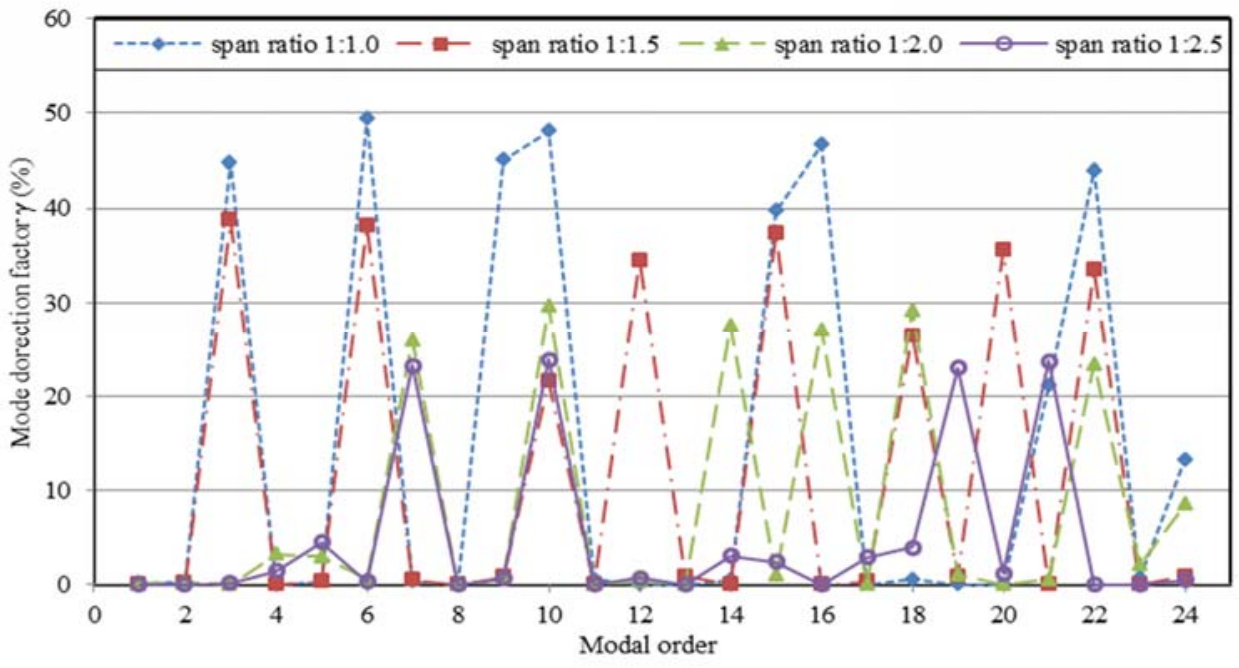

FIGURE VI. VALUES AND COMPARISON OF MODE DIRECTION FACTOR $\Gamma$ 


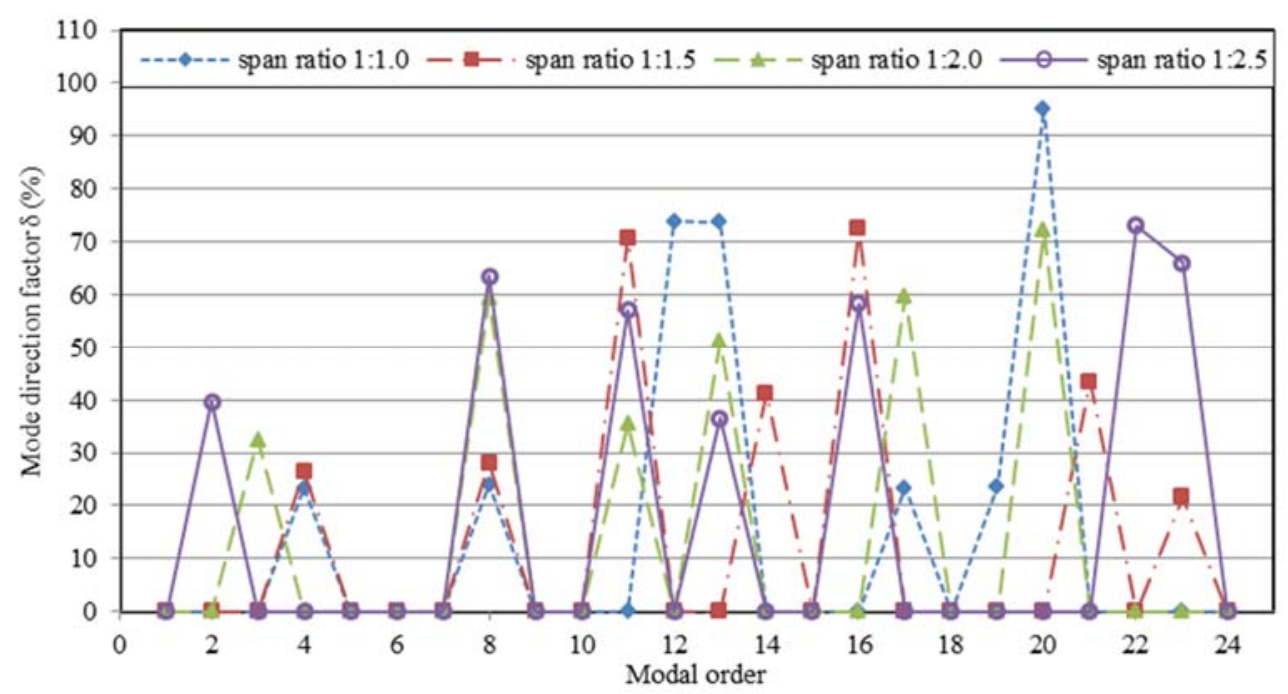

FIGURE VII. VALUES AND COMPARISON OF MODE DIRECTION FACTOR $\Delta$

As can be seen from FIGURE IV that, the values of mode direction ratio $\alpha$ are bigger and appears more than those of other mode direction ratios in mode orders for all the models with different span ratios. So the vertical bending mode should be taken fully consideration for curved girder bridges with span ratios of $1: 1$ to $1: 2.5$.

FIGURE $\mathrm{V}$ states that the peak value of mode direction ratio $\gamma$ decreases slightly as the span ratio diminishes, but is all larger than $50 \%$. On another hands, the ratio is lateral bending mode directional ratios, reflecting lighter destruction, can be considered for reference in design of curved box-girder bridges.

FIGURE VI shows that the value of mode direction ratio $\beta$ decreases as the span ratio decreases, but the value is up to $50 \%$. When the span ratio is $1: 2.5$, the maximum of $\beta$ is not more than $25 \%$. So the notion mode can be taken no account of for curved box-girder bridges when the span ratio is less than $1: 2$.

FIGURE VII indicates that the mode direction ratio $\delta$ appears in higher orders of mode, and the higher the peak, the higher the order. So the influence of longitudinal shift directional ratio should be considered on higher orders of modes.

The principal mode is defined as the mode direction ratio is more than 0.5 according to reference [10]. Four mode direction ratios were defined in this paper, if the value of one ratio is greater than 0.25 , which is average of the above four ratios, the mode in the direction is the principal mode. Further, the $n$th order of modal occurrence is called the $n$th mode of vibration. For example, the first occurrence order of modal, which principal mode is vertical bending mode, is called the 1 st order of vertical bending. The other principal modes are named similarly.

FIGURE VIII to FIGURE XI are the orders of mode direction ratios for all models with different span ratios.

FIGURE VIII shows that the principal mode of vertical bending appears more, the same rule exits in models with different span ratios, and almost 17 18 orders are present in total 24 orders. This means the vertical bending modes should be focused on in design of curved box-girder bridges.

FIGURE X presents that the order of torsion principal mode appears later in all the modal orders with decreasing span ratio; and the torsion principal mode will disappear in models with span ratios smaller than $1: 2.5$.

As can be seen from FIGURE IX and FIGURE XI that, the mode direction ratios of transverse bending and longitudinal traverse appears earlier in low orders with smaller span ratios (the ratio arises later as horizontal coordinate is greater on the same vertical coordinate). This rule will be disturbed at larger mode orders. So, the modes of transverse bending and longitudinal traverse are advised to be considered at lower modals for curved box-girder bridges with smaller span ratios in design.

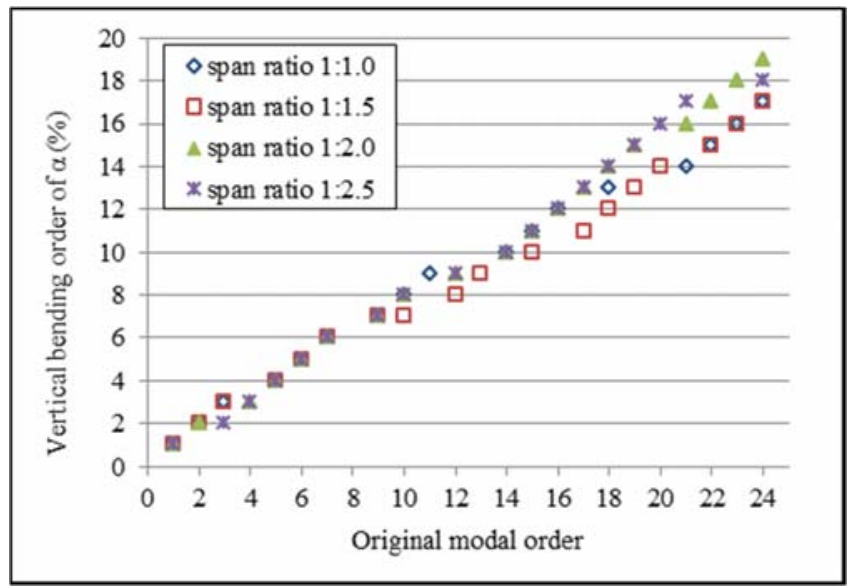

FIGURE VIII. VERTICAL BENDING ORDER OF MODE DIRECTION FACTOR A 


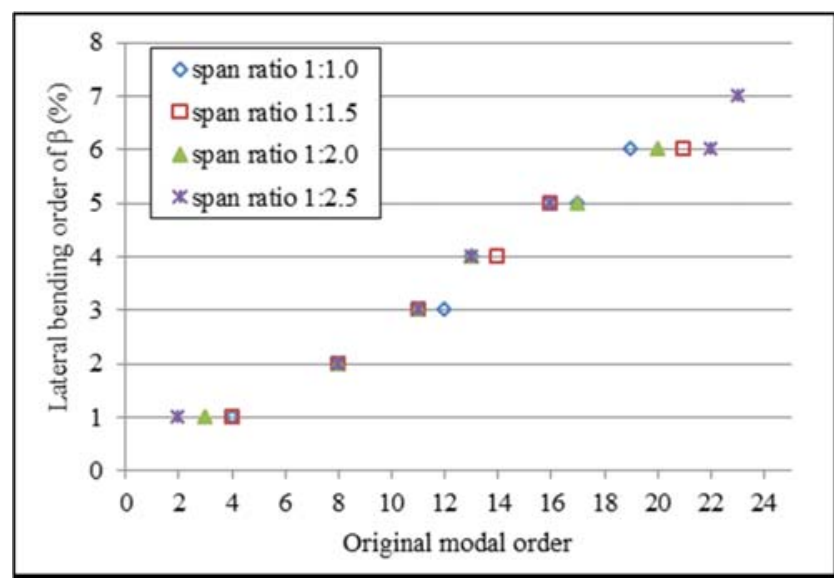

FIGURE IX. LATERAL BENDING ORDER OF MODE DIRECTION FACTOR B

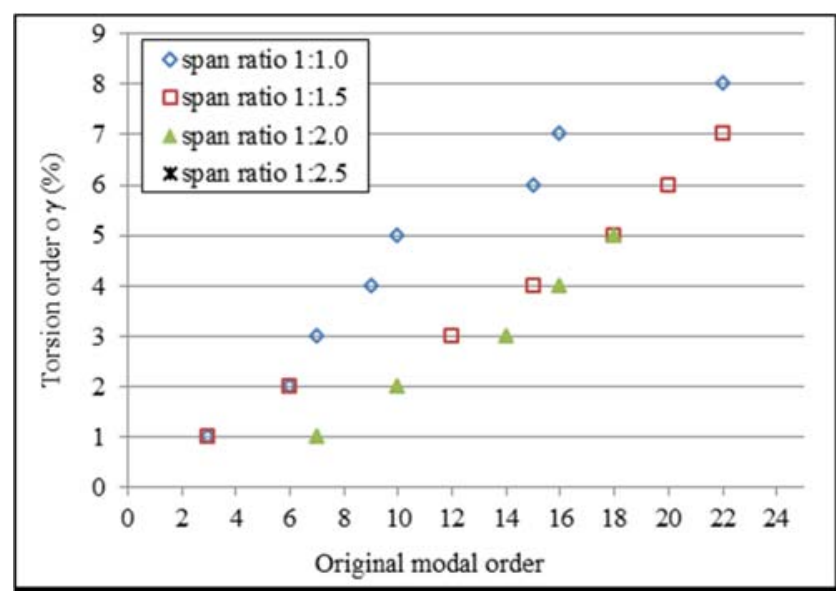

FIGURE X. TORSION ORDER OF MODE DIRECTION FACTOR $\Gamma$

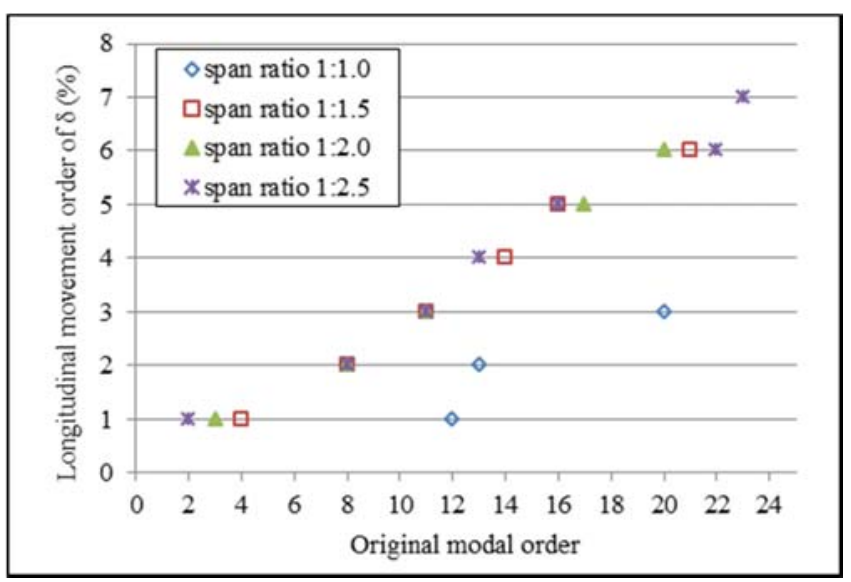

FIGURE XI. LONGITUDINAL MOVEMENT ORDER OF MODE DIRECTION FACTOR $\Delta$

\section{CONCLUSIONS}

The vibration mode of curved box-girder bridge was decomposed into 4 mode direction factors by energy ratios. Four curved box-girder bridge models with two spans were built taking span ratio as parameter. The modal characteristics and the mode direction factors were analyzed along with varying span ratio, some advice were concluded for design of curved box-girder bridges with two spans as follows.

a) Stiffness increases slower than mass when span ratio decreases for curved box-girder bridges. That is, the smaller the span ratio, the slender the stiffness.

b) The vertical bending mode must be considered when span ratio is between 1:1 and 1:2.5 for curved box-girder bridges.

c) The mode direction factor of transverse bending $\beta$ decreases slightly as span ratio decreases, but the destruction caused is mildly.

d) The mode direction factor of torsion $\gamma$ decreases with span ratio diminishing, and the ratio can be neglected when span ration is less than 1:2 for curved box-girder bridges.

e) The mode direction factor of longitudinal movement $\delta$ has a peak value in higher order mode for smaller span ratios. So the higher order modals should be taken into account in design.

\section{ACKNOWLEDGMENT}

The authors thank National Natural Science Foundation of China (U1304515), the authors thank anonymous reviewers for valuable comments and suggestions.

\section{REFERENCES}

[1] Zhu Dongsheng, Liu Shizhong, Yu Lusong, "Research on seismic response of curved girder bridges," China Journal of Highway and Transport, Vol. 15, pp. 42-48, June 2002. (in Chinese)

[2] Zhou Xuhong, Dai Peng, Di Jin, "Nonlinear analysis of curved prestressed concrete box girder bridge with seismic isolation system," China Journal of Highway and Transport, Vol. 21, pp. 65-71, February 2002. (in Chinese)

[3] Xu Xun, Wei Xing, Liu Dejun, et al,. "Analysis for static and dynamic characteristics of flat curved box beam," Journal of Highway and Transportation Research and Development, Vol. 24, pp. 60-65, December, 2007. (in Chinese)

[4] Zhao Qing, Xiao Zhuo, "Analysis on dynamic characteristic parameters of a curved box-girder bridge," World Earthquake Engineering, Vol. 26, pp. 197-201, January, 2013. (in Chinese)

[5] Xie Guanmo. Mechanics of Vibration, Beijing: National Defence Industry Press, 2011. (in Chinese)

[6] Yin Lei, "Mode classification and quantification research of engineering structure,” Yellow River, Vol. 35, pp. 96-97, April 2013. (in Chinese)

[7] Wu Zeyu, Wang Dongwei, "Mode-based concept design of engineering structure," Earthquake Resistant Engineering and Retrofitting, Vol. 34, pp. 42-45, August 2012. (in Chinese)

[8] Xing Shiling, Ye Jianshu, Yu Bo, "A method to determine main direction of a curved bridge's mode shape," Journal of Highway and Transportation Research and Development, Vol. 27, pp. 79-84, February 2010. (in Chinese)

[9] Wu Dingyi, Deng Cibing, "Discussion on fixing rational shaking number by valid quality methods," Shanxi Architecture, Vol. 33, pp. 5758, June 2007. (in Chinese)

[10] Wang Fuzhi, Wang Yiqun, Deng Xiaoxiang, "Selection of mode num and determination of torsional mode," Journal of Tianjin University of Technology, Vol. 23, pp. 81-85, June 2005. (in Chinese)

[11] "Guidelines for Seismic Design of Highway Bridges (JTG/T B02-012008)," Ministry of Transport of the People's Republic of China. 2008. (in Chinese) 